\title{
Estudio comparativo de los estilos de apego adulto en un grupo de mujeres con y sin diagnóstico de depresión
}

\section{Comparative study of adult attachment styles in a group of women with and without a diagnosis of depression}

\author{
Lusmenia Garrido \\ Universidad Católica del Maule, Chile \\ Mónica Guzmán \\ Universidad Católica del Norte, Chile \\ María Pía Santelices \\ Pontificia Universidad Católica de Chile \\ Verónica Vitriol \\ Universidad de Talca, Hospital de Curicó, Chile \\ Estrella Baeza \\ Universidad Católica del Maule, Chile
}

(Rec: 1 febrero 2013 / Acept: 28 septiembre 2015)

\begin{abstract}
Resumen
Numerosos estudios han asociado los estilos de apego inseguros a distintas formas de psicopatología, entre ellas la depresión, en diversas etapas del ciclo vital. Esta investigación tuvo como objetivo describir el estilo de apego de un grupo de pacientes de sexo femenino, diagnosticadas con depresión. Se estudió a un grupo de 28 pacientes, comparándolos con un grupo de 28 mujeres adultas que no presentan la enfermedad, igualando a las participantes en relación a la edad. Los resultados mostraron diferencias significativas en los estilos de apego de ambos grupos. Las pacientes diagnosticadas con depresión presentan, en su mayoría, estilos de apego inseguros (39.2\%) e inclasificables (32.1\%). Por su parte, el grupo de personas sin depresión presentan en su gran mayoría $(85.7 \%)$ un estilo de apego seguro.

Palabras clave: depresión, estilos de apego, apego adulto y psicopatología.
\end{abstract}

\begin{abstract}
Several studies have linked the insecure attachment styles to different types of psychopathologies, i.e. depression, at diverse stages of the life cycle. This research aimed to describe the attachment style for a group of female patients diagnosed with depression. A group of 28 patients was analyzed and compared with a group of 28 female adults not presenting the psychopathology and with similar age range. Results show significant differences on the attachment style in within these two groups. The patients diagnosed with depression have, mainly, insecure attachment styles $(39.2 \%)$ and cannot classify $(32.1 \%)$. On the other side, the majority of the group of people without depression $(85.7 \%)$ has a secure attachment style.

Keywords: depression, attachment styles, adult attachment, psychopathology.
\end{abstract}

* Correspondencia: Lusmenia Garrido, Dirección: Avenida San Miguel 3605, Talca, Chile. Teléfono: (56-71) 203100, E-mail: lgarrido@ucm.cl La elaboración de este artículo contó con el financiamiento del Proyecto Interno No 8.1.4.11. de la Universidad Católica del Maule y el Proyecto FONDECYT 1040760. 


\section{Introducción}

Desde los inicios de la teoría del apego (Bowlby, 1969, 1973,1980 y 1986) hasta la actualidad, ha habido un desarrollo notable de investigación científica en el área y una gran diversificación en las distintas temáticas que se abordan desde esta perspectiva. La presente investigación, tuvo como objetivo evaluar el estilo de apego adulto en un grupo de mujeres con depresión y comparar éste con una muestra no clínica.

El apego adulto se refiere a la tendencia estable de un ser humano de hacer esfuerzos sustanciales para buscar y mantener proximidad y contacto con uno o unos pocos individuos específicos, quienes proveen el potencial subjetivo para la seguridad y protección física y/o psicológica (Berman y Sperling, 1994). Esta tendencia es regulada por modelos operantes internos de apego, los cuales son esquemas cognitivo-afectivo-motivacionales construidos desde la experiencia del individuo en su mundo interpersonal. Para Bowlby (1973), cada persona construye modelos operantes del mundo, en que una característica clave es su idea de quiénes son sus figuras de apego y cómo responden, $\mathrm{y}$, también, modelos operantes de sí mismo, que contienen la noción de cuán aceptable aparecen ante sus figuras de apego; ambos modelos le ayudan al sujeto a percibir sucesos, pronosticar lo que ocurrirá y construir planes. Este concepto es la piedra angular de la evaluación del apego en el adulto.

\section{Evaluación del apego adulto}

Las primeras mediciones de los estilos de apego surgieron en el contexto de la evaluación de la relación en díadas madre-hijo, desarrollando a partir de esto tres clasificaciones del apego en niños (Ainsworth, Blehar, Waters y Wall 1978), que son el estilo seguro, evitativo y ambivalente, al que se sumó posteriormente el estilo desorganizado (Main y Solomon, 1990). Posteriormente, las investigaciones se extrapolaron a otras etapas del desarrollo, iniciándose una importante tradición en la medición del apego adulto, lo que ha implicado complejidades y desafíos a los investigadores, ya que los estudios han surgido desde distintas áreas de la psicología y, por lo mismo, han realizado énfasis diversos en la conceptualización del apego adulto, en la forma de evaluar, en el foco de la evaluación, en el tipo de instrumentos utilizados, etc. (Bartholomew, 1994; Bifulco, 2002; Feeney, Noller y Hanrahan, 1994; Griffin y Bartholomew, 1994; Harris, 2002; Hazan y Shaver, 1994; Jacobvitz, Curran y Moller, 2002; Mikulincer y Shaver, 2007; Roisman et al.,
2007; Shaver y Mikulincer, 2002; Stein, Jacobs, Ferguson, Allen y Fonagy, 1998).

Específicamente en Chile, Martínez y Santelices (2005) señalaban que no existía una tradición en medición o investigación del apego en adultos, sumado a una escasa literatura nacional en el tema. No obstante, existe un avance paulatino en los últimos años, aumentando los instrumentos validados, investigaciones y publicaciones en el área (Garrido, 2013).

$\mathrm{Al}$ Parental Bonding Instrument, validado el año 2001 en Chile (Melis et al., 2001), se han sumado otros instrumentos. Martínez y Núñez (2007) publicaron la adaptación de la Entrevista de Prototipos de Apego Adulto (EPAA). Garrido, Santelices, Pierrehumbert y Armijo (2009) publicaron la validación chilena del Camir: Cartes, Modèles Individuels de Relation (Pierrehumbert, Karmaniola, Sieye, Meister, Miljkovitc y Halfon, 1996), instrumento de autoinforme que presenta una adecuada confiabilidad y que ya cuenta, además, con un manual que incluye sus normas (Garrido, 2013). También se encuentra validado el Experiences in Close Relationships (ECR), instrumento de autoinforme de apego en relaciones románticas en adultos (Spencer, Guzmán, Fresno y Ramos, 2013) y su versión reducida (Spencer, Alonso-Arbiol y Fresno, 2013).

En el ámbito de la investigación, en los últimos años se han publicado interesantes estudios en apego adulto en nuestro país, tanto en el sector de la salud física (Santelices, Garrido, Fuentes, Guzmán y Lizano, 2009), como mental (Lecannelier et al., 2009; Farkas, Santelices, Aracena y Pinedo, 2008; Quezada y Santelices, 2010; Rivera, Cruz y Muñoz, 2011; Santelices, Guzmán y Garrido, 2011) y en factores asociados como la sensibilidad del adulto (Santelices, Carvacho, Farkas, León, Galleguillos y Himmel, 2012).

\section{Depresión}

La depresión es una de las patologías mentales que se diagnostica más comúnmente entre los adultos, siendo una de las principales causas de enfermedad a través del mundo entero. Estudios epidemiológicos evidencian no sólo una alta prevalencia de los desórdenes depresivos, sino también edades tempranas de inicio, alta cronicidad y discapacidad en los diversos roles que desempeñan las personas (Richards, 2011). Esto se complejiza aún más, si se considera que el pronóstico de vida de los trastornos del estado de ánimo se encuentra especialmente perjudicado por la alta suicidalidad que se presenta en la fase depresiva; la frecuencia de suicidios en las depresiones fluctúa entre un 7 y un 10\% (Heerlein, 2000), constituyéndose como uno de los principales factores de riesgo para el intento de suicidio (Davidson, 
Wingate, Grant, Judah y Mills, 2011; Preti, Tondo, Sisti, Rocchi y De Girolamo, 2010). Así, más de la mitad de las muertes por suicidio se deben a depresión y es reconocido que las personas con esta patología experimentan el doble de la mortalidad que la población general y sufren mayores limitaciones en su funcionamiento psicosocial que aquellas con enfermedades físicas crónicas como hipertensión y diabetes (Retamal et al., 2004).

Específicamente en Chile, la depresión es un problema de primera importancia para la salud pública, presentando una prevalencia de 7.5 a $10 \%$ en la población adulta (Retamal et al., 2004). Las últimas dos encuestas nacionales en salud (Ministerio de Salud [MINSAL], 2003 y 2010) reportan una prevalencia de síntomas depresivos en el último año de $17.5 \%$ y $17.2 \%$ respectivamente. En una reciente investigación en universitarios que consultan a un servicio de salud estudiantil, los trastornos del ánimo correspondieron a la segunda mayor incidencia encontrada, con un $22.8 \%$ (Micin y Bagladi, 2011).

Los costos asociados con la depresión son elevados (Retamal et al., 2004), incluyendo aspectos interpersonales, personales, sociales y económicos (Richards, 2011). Particularmente en Chile, la depresión se constituye en la segunda causa de AVISA (años de vida saludable perdidos por discapacidad y muerte prematura) en mujeres (MINSAL, 1999) y, además, es responsable de un tercio de los días de trabajo perdidos debido a enfermedad, concentrando más del $40 \%$ de las licencias médicas (Retamal et al., 2004). Todos los elementos señalados anteriormente hicieron que el abordaje masivo e integral de los trastornos depresivos se convirtiera en un desafío y prioridad a nivel nacional, justificando la presencia de intervenciones focalizadas en el área (MINSAL, 1999). Es así como durante la década anterior se generaron diversas estrategias para mejorar el tratamiento de la depresión, como su incorporación en las Garantías Explícitas en Salud (GES), la expansión del Programa Integral de Salud Mental en Atención Primaria que incluye depresión, la incorporación de psicólogos en atención primaria, entre otras; para esta década, la Estrategia Nacional de Salud 2011-2020 (MINSAL, 2011) propone aumentar la cobertura de los equipos especializados en salud mental y mejorar la calidad de las intervenciones.

\section{Apego y depresión}

A partir de la década de los 90 comienzan a aparecer una serie de investigaciones que relacionan los estilos de apego con distintas formas de psicopatología, tanto en la niñez, como en la adolescencia y adultez, las que fueron rigurosamente sistematizadas y analizadas en el Handbook of Attachment (Greenberg, 1999; Dozier, Stovall y Albus, 1999).

Específicamente en el ámbito de la depresión y su relación con los estilos de apego, es posible encontrar investigaciones desde la década de los 90 (Carnelley, Pietromonaco y Jaffe, 1994; Hortacsu, Cesur y Oral, 1993); sin embargo, es a partir del año 2000 hasta la actualidad cuando se ha producido un aumento considerable en el interés por esta temática, tanto en la niñez (Capozzi et al., 2008; Graham, 2000; Kerns, Brumariu y Seibert, 2011; Muris, Mayer y Meesters, 2000; Roelofs, Meesters, Huurne, Bamelis y Muris, 2006), adolescencia (Figueiredo, Bifulco, Pacheco, Costa y Magarinho, 2006; Gamble y Roberts, 2005; González y Méndez, 2006; Ivarsson, Granqvist, Gillberg y Broberg, 2010; Nishikawa, Hägglöf y Sundbom, 2010; Ruijten, Roelofs y Rood, 2011), adultez (Altin y Terzi, 2010; Armour, Elklit y Shevlin, 2011; Bifulco et al., 2006; Bifulco, Moran, Ball y Bernazzani, 2002a; Bifulco, Moran, Ball y Lillie, 2002b; Conradi y De Jonge, 2009; Cooley, Van Buren y Cole, 2010; Evans y Wertheim, 2005; Figueiredo, Bifulco, Pacheco, Costa y Magarinho, 2006; Hankin, 2005; Kuscu et al., 2008; Li, Li y Dai, 2008; Liu, Nagata, Shono y Kitamura, 2009; Mak, Simpson y Rholes, 2010; Meredith, Strong y Feeney, 2007; Miljkovitch, Pierrehumbert, Karmaniola, Bader y Halfon, 2005; Monk, Leight y Fang, 2008; Myhr, Sookman y Pinard, 2004; Permuy, Merino y Fernández-Rey, 2009; Pesonen, Räikkönen, Strandberg, Keltikangas-Järvinen y Järvenpää, 2004; Simonelli, Ray y Pincus, 2004; Strodl y Noller, 2003; Surcinelli, Rossi, Montebarocci y Baldaro, 2010; Tasca et al., 2009; Whiffen, Kerr y Kallos-Lilly, 2005; Wilkinson y Mulcahy, 2010; Zaghdoudi, Moubarkim, Hlayem, Bechir y Labbane, 2009) y adultos mayores (Besser y Priel, 2008).

En la etapa de la niñez, Muris et al. (2000) realizaron una investigación que tuvo como propósito explorar la relación entre estilo de apego y niveles de ansiedad y depresión. Los autores reportan que los niños con estilos de apego inseguros mostraron elevados niveles de ansiedad y depresión, en comparación con niños con estilo de apego seguro. No obstante, no se hallaron diferencias significativas entre los niños con apegos evitativos y ambivalentes. Por otra parte, Roelofs et al. (2006) investigaron las relaciones entre estilo de apego, pautas de crianza parental y problemas internalizantes y externalizantes en niños, en una muestra no clínica; específicamente con respecto a la relación entre estilo de apego y síntomas internalizantes y externalizantes, el apego inseguro fue asociado con puntajes más altos en ansiedad, depresión y agresión. Sin embargo, el estilo de apego juega un rol menos prominente que las conductas negativas de crianza. En una investigación en niños con dificultades de aprendizaje se encontró que las conductas internalizadoras aparecieron 
asociadas con dimensiones de apego rechazante en el padre y apego preocupado en la madre (Capozzi et al., 2008). Por último, Kerns et al. (2011) investigaron cómo el apego está relacionado con la parentalidad y la depresión; a nivel general, niños con apego seguro reportaron menores niveles de síntomas depresivos. En tanto, niños con apego ambivalente reportan más síntomas depresivos, aunque esta relación no es significativa después de controlar el sexo, ya que son las niñas más que los niños quienes reportan mayor sintomatología. Por último, niños desorganizados reportan niveles más altos de síntomas depresivos, a pesar de que esta asociación deja de ser significativa al controlar el status familiar.

En el periodo de la adolescencia, las investigaciones también dan cuenta de una asociación entre estilos de apego inseguros y desórdenes depresivos. Ivarsson et al. (2010) realizaron un estudio con adolescentes, incluyendo trastorno depresivo y/o trastorno obsesivo compulsivo y controles. Mientras alrededor de la mitad de los adolescentes de la población general presentaban un estilo de apego seguro, para el caso de adolescentes con depresión, solamente un $8 \%$ de ellos presentó apego seguro. El estilo inseguro predominante fue el estilo evitante, con una baja presencia del estilo preocupado. Por otra parte, un estudio que examinó las relaciones entre apego, autoconcepto y problemas de salud mental, reporta que los adolescentes con apego inseguro tuvieron niveles más altos de problemas internalizadores y sociales que aquellos que presentaban estilo seguro (Nishikawa et al. 2010).

En cuanto a las investigaciones en apego adulto y depresión, a nivel general también existe una clara asociación entre los estilos inseguros de apego, la presencia de sintomatología y trastornos depresivos. Sin embargo, no existe acuerdo aún, en términos de cuál estilo predomina en personas con depresión, ya que se aprecian diferencias importantes en los resultados de las investigaciones, sumado a la utilización de distintos instrumentos de evaluación y categorías, lo que en ocasiones dificulta poder realizar comparaciones adecuadas (Dozier, Stovall y Albus, 1999; Mikulincer y Shaver, 2007). Considerando la mayoría de los estudios en este ámbito, algunas de las clasificaciones de mayor utilización son las siguientes: la propuesta por Main (2000), que distingue el estilo seguro-autónomo (secure-autonomous), rechazante (dismissing), preocupado (preoccupied) y no resuelto (unresolved); otra clasificación es la de Bartholomew y Horowitz (1991), que propone los estilos seguro (secure), preocupado (preoccupied), temeroso (fearful) y rechazante (dismissing); la propuesta de Bifulco et al. (2002a), que clasifica a los sujetos en estilo seguro (clearly standard or secure), enredado (enmeshed), temeroso (fearful), rechazante rabioso (angry-dismissive) y retraído (withdrawn) y, por último, la tipología de Hazan y Shaver (1987), de estilo seguro (secure), evitativo (avoidant) y ansioso-ambivalente (anxious/ambivalent) ${ }^{1}$.

Una línea de investigación en el ámbito adulto, tiene que ver con la depresión asociada al periodo perinatal o al periodo posparto y su relación con los estilos de apego. En una investigación realizada por Monk et al. (2008), evaluaron a 186 mujeres embarazadas, hallando que mujeres con un estilo de apego temeroso (Bartholomew y Horowitz, 1991), tuvieron más probabilidad de estar deprimidas durante el embarazo. Además, bajos puntajes en seguridad del apego implican un mayor riesgo para la depresión posparto. En la misma línea, Figueiredo et al. (2006) compararon la experiencia de embarazo en adolescentes y adultas; uno de los principales predictores de la depresión fue el estilo de apego enredado (Bifulco et al., 2002), caracterizado por un elevado deseo de compañía, baja autoconfianza y elevado miedo de separación.

Específicamente en la depresión posparto, Kuscu et al. (2008) encontraron correlaciones positivas significativas entre los puntajes totales de la Escala de Depresión Posparto de Edimburgo y el estilo de apego ambivalente y evitativo (Hazan y Shaver, 1987); por el contrario, el apego seguro mostró una relación inversa. Por último, en una investigación de madres con y sin diagnóstico de depresión, las primeras reportaron menor seguridad en el apego y mayor presencia de estilos temerosos y preocupados (Bartholomew y Horowitz, 1991); en tanto, madres clínicamente deprimidas fueron significativamente más inseguras en su estilo de apego que el grupo de madres no deprimidas (Wilkinson y Mulcahy, 2010).

Por otro lado, diversas investigaciones han relacionado estilos de apego adulto con depresión o sintomatología ansiosadepresiva en muestras no clínicas. A nivel más general, el apego inseguro ha sido vinculado con más altos niveles de depresión (Liu et al., 2009; Surcinelli et al., 2010). En las investigaciones que han utilizado el modelo de Bartholomew y Horowitz (1991), se ha encontrado sistemáticamente que los participantes que presentan estilos de apego preocupado y temeroso reportan más sintomatología depresiva en comparación con el estilo de apego seguro y rechazante y con el grupo control (Carnelley et al., 1994; Cooley et al., 2010; Permuy et al., 2009; Simonelli et al., 2004; Surcinelli et al., 2010; Van Buren y Cooley, 2002). Tanto en el estilo de apego preocupado como temeroso se presenta un modelo negativo de sí mismo; en el caso del estilo temeroso se acompaña de un modelo negativo de los otros, no así en el estilo preocupado, en que el modelo de los otros

\footnotetext{
Se explicitan, en cursiva, las palabras en inglés de los autores originales de cada clasificación; en el caso de Bifulco et al. (2002a), la traducción es de los autores.
} 
es positivo (Bartholomew y Horowitz, 1991). Por último, en una investigación de Santelices et al. (2011), que utilizó las categorías seguro, preocupado y rechazante, se encontró una correlación significativa y directa entre sintomatología ansiosa-depresiva y estilos inseguros; sin embargo, el mayor nivel de desajuste lo presentó el estilo preocupado.

Algo similar a lo reportado en población general ocurre en las investigaciones con muestras clínicas, en el sentido que los participantes que presentan depresión reportan mayor inseguridad en el estilo de apego (Evans y Wertheim, 2005; Myhr et al., 2004; Strodl y Noller, 2003; Zaghdoudi et al., 2009). Sin embargo, se aprecian algunos énfasis distintos e interesantes en este tipo de población. En las investigaciones que utilizan la tipología de Bartholomew y Horowitz (1991), hay dos tendencias principales; por un lado, Haaga et al. (2002) señalan que tanto el apego inseguro temeroso como preocupado se constituyen en un factor de vulnerabilidad estable para la depresión. No obstante, otras investigaciones han reportado que el estatus de la depresión se relaciona específicamente con el estilo de apego temeroso (Carnelley et al., 1994). En esta línea, Conradi y De Jonge (2009) examinaron una muestra de pacientes con una depresión de larga duración, concluyendo que los sujetos con apego temeroso constituyen una categoría especialmente vulnerable, reportando un peor curso de la depresión, mayor utilización de antidepresivos, más episodios depresivos anteriores y síntomas residuales. En una investigación que relacionó estilos de apego adulto y depresión, en un grupo de pacientes con dolor crónico, se halló la misma relación, es decir, los participantes que presentaron un estilo de apego temeroso tuvieron las más altas tasas de depresión comparado a los demás estilos (Meredith et al., 2007). Por último, Bifulco et al. (2002a, 2002b y 2006) aportan una distinción interesante, ya que establecen distintos niveles en la inseguridad del apego, siendo ésta ligera, moderada o marcadamente insegura. En este sentido, estilos moderados o marcadamente inseguros estuvieron más altamente relacionados con la depresión clínica (Bifulco et al., 2002a y 2006), con poca discriminación entre los distintos tipos de apego inseguro; no obstante, los estilos moderado o marcadamente enredado, temeroso o rechazante rabioso, se relacionaron en mayor medida con el desorden y se asociaron a otros factores de vulnerabilidad a la depresión. Armour et al. (2011) realizaron un estudio en una muestra con víctimas de traumas, hallando que estilos preocupados y temerosos tuvieron puntajes promedios más altos en depresión $\mathrm{y}$ ansiedad que el estilo seguro.

\section{Método}

\section{Participantes}

Se evaluó un total de 56 mujeres, 28 de las cuales eran pacientes diagnosticadas con depresión y 28 pertenecientes a población no clínica (sin el diagnóstico de depresión), provenientes de la Región Metropolitana y del Maule de Chile. En el caso de la muestra clínica, ésta se extrajo del grupo de pacientes que acudían al programa de depresión del Hospital de Curicó, mientras que la muestra no clínica se extrajo de la base de datos utilizada para el estudio de validación chilena del Camir (Garrido et al., 2009), igualando a las participantes por edad. La edad de la muestra se ubicó entre los 18 y los 51 años $(M=31.1 ; D E=9.98)$.

Cabe mencionar que las participantes aceptaron participar voluntariamente en la investigación, previa firma del consentimiento informado. Para la realización del estudio se contó con la aprobación de los comités de ética de las instituciones involucradas.

\section{Instrumentos}

Para la evaluación de la depresión se utilizó una entrevista semi-estructurada, teniendo como base los criterios de la Clasificación Internacional de Enfermedades CIE 10 (Organización Mundial de la Salud [OMS], 1999), realizando el diagnóstico el médico psiquiatra del Hospital de Curicó. La totalidad de las pacientes presentaba el diagnóstico de episodio depresivo grave, de acuerdo a los criterios señalados.

Para la evaluación del apego se utilizó el Camir, desarrollado por Pierrehumbert et al. (1996) y validado en Chile por Garrido et al. (2009). Investiga las evaluaciones de los participantes de experiencias pasadas y presentes, con la familia de origen y con las figuras actuales de apego respectivamente, sus interpretaciones personales de las actitudes de sus padres durante la niñez y el impacto que éstas tuvieron y sus concepciones del funcionamiento familiar.

Este instrumento consta de 71 ítems, repartidos en 13 escalas: Interferencia parental (IP), Preocupación familiar (PF), Resentimiento de infantilización (RI), Apoyo parental (AP), Apoyo familiar (AF), Reconocimiento de apoyo (RA), Indisponibilidad parental (IP2), Distancia familiar (DF), Resentimiento de rechazo (RR), Traumatismo parental (TP), Bloqueo de recuerdos (BR), Dimisión parental (DP) 
y Valoración de la jerarquía (VJ). Las escalas IP, PF y RI dan cuenta del apego preocupado; AP, AF y RA, del apego seguro; IP2, DF y RR, del apego rechazante; TP y BR se relacionan con la no resolución del apego; y DP y VJ con la estructuración del medio familiar.

La validación de Camir en Chile encontró una fiabilidad adecuada, con valores alfa entre 0.72 y 0.84 , salvo en las escalas IP $(\alpha=.54)$, DF $(\alpha=.33)$, DP $(\alpha=.39)$ y VJ $(\alpha=.33)$ (Garrido et al., 2009).

El formato de respuesta del instrumento, en una primera etapa, es de tipo Likert de 1 a 5 (desde muy verdadero a muy falso). En una segunda etapa se agrega el procedimiento Q-Sort, en que se obliga al sujeto a dejar una cantidad específica de cartas en cada categoría (desde muy verdadero, verdadero, ni verdadero ni falso, falso a muy falso). Los puntajes escalares de Camir se calculan en base a las medias de los puntajes de los ítems que las componen. De esta manera, todas las escalas pueden obtener puntajes que fluctúan entre 1 y 5 puntos, donde un mayor puntaje indica mayor presencia de la dimensión medida. A fin de obtener el estilo de apego predominante de cada persona, se combinan los puntajes escalares correspondientes a cada uno de los patrones de apego (seguro, preocupado y rechazante), obteniéndose un perfil de cada sujeto. Dicho perfil es comparado, mediante correlación, con un patrón estándar de respuesta representativo de cada estilo (prototipo), con puntajes que pueden fluctuar entre - 1 y 1 , donde un mayor puntaje indica una mayor correspondencia a un patrón de apego. Con base en esos datos se calcula el puntaje estandarizado con el cual se puede clasificar a cada sujeto según el patrón de apego predominante, comparando los puntajes obtenidos en cada escala y asignándolos a la categoría de la escala donde obtienes un puntaje mayor. Cuando hay más de un prototipo que presenta puntajes muy altos, se asume que existe un estilo de apego inclasificable (para una descripción detallada de cada uno de los prototipos y sus características, ver Garrido, 2013).

\section{Resultados}

En la Tabla 1 puede observarse la estadística descriptiva para las escalas del Camir. Para la muestra total, los puntajes más elevados se observan en las escalas de valoración de jerarquía $(M=3.57)$ y preocupación familiar $(M=3.56)$.

Similar tendencia se observa en la muestra clínica, con puntajes más elevados en valoración de la jerarquía $(M=$ 4.40) y preocupación familiar $(M=3.92)$. En el caso de la muestra sin depresión, los puntajes más altos se observan también en la escala de interferencia parental $(M=3.77)$ y reconocimiento de apoyo $(M=3.52)$.

No se encontraron asociaciones significativas entre la edad de las participantes y los puntajes obtenidos en las escalas del Camir $(p>.05)$, ni relación con los puntajes en cada uno de los tipos de apego $(p>.05)$.

Tabla 1. Medidas descriptivas para las escalas Camir.

\begin{tabular}{lccc}
\hline ESCALA & Total & Sin depresión & Con depresión \\
$M(D E)$ & $M(D E)$ & $3.77(0.40)$ & $3.09(0.57)$ \\
\hline Interferencia Parental & $M(D E)$ & $3.20(0.59)$ & $3.92(0.51)$ \\
Preocupación Familiar & $3.43(0.60)$ & $2.30(0.64)$ & $3.09(0.85)$ \\
Resentimiento de Infantilización & $3.56(0.65)$ & $3.41(0.59)$ & $3.08(0.95)$ \\
Apoyo Parental & $2.70(0.84)$ & $2.36(0.66)$ & $3.45(0.94)$ \\
Apoyo Familiar & $3.25(0.80)$ & $3.52(0.68)$ & $3.34(0.91)$ \\
Reconocimiento de Apoyo & $2.91(0.98)$ & $3.31(0.47)$ & $3.15(0.91)$ \\
Indisponibilidad Parental & $3.43(0.80)$ & $2.39(0.93)$ & $3.27(0.77)$ \\
Distancia Familiar & $3.23(0.72)$ & $1.94(0.71)$ & $3.04(1.11)$ \\
Resentimiento de Rechazo & $2.83(0.95)$ & $2.48(0.42)$ & $2.92(1.08)$ \\
Traumatismo Parental & $2.49(1.08)$ & $2.84(0.68)$ & $2.82(0.86)$ \\
Bloqueo de Recuerdos & $2.70(0.84)$ & $3.21(0.65)$ & $2.36(0.83)$ \\
Dimisión Parental & $2.83(0.77)$ & $2.75(0.52)$ & $4.40(0.44)$ \\
Valoración de Jerarquía & $2.79(0.86)$ & $3.57(0.96)$ & \\
\hline
\end{tabular}

Nota $. ~ N=56$. Muestra no clínica $=28$; Muestra con depresión $=28$. Los puntajes representan la media de la escala, en un rango que fluctúa de 1 a 5 , donde 5 representa una mayor identificación del sujeto con los ítems de la escala. 
Tabla 2. Clasificación obtenida según el tipo de apego dominante, en base a norma original o puntajes muestrales.

\begin{tabular}{lcc}
\hline \multicolumn{1}{c}{$\begin{array}{c}\text { ESTILO DE } \\
\text { APEGO }\end{array}$} & $\begin{array}{c}\text { SIN } \\
\text { DEPRESIÓN } \\
\mathrm{n}(\%)\end{array}$ & $\begin{array}{c}\text { CON } \\
\text { DEPRESIÓN } \\
\mathrm{n}(\%)\end{array}$ \\
\hline Apego Seguro & $24(85.7 \%)$ & $8(28.6 \%)$ \\
Apego Rechazante & $1(3.6 \%)$ & $2(7.1 \%)$ \\
Apego Preocupado & $3(10.7 \%)$ & $9(32.1 \%)$ \\
Inclasificable & - & $9(32.1 \%)$ \\
\hline
\end{tabular}

Nota. $N=56$

La distribución por tipo de apego se observa en la Tabla 2. En la muestra sin depresión, el mayor porcentaje $(85.7 \%)$ de las participantes posee un estilo de apego seguro. Por su parte, en la muestra diagnosticada con depresión, los mayores porcentajes corresponden a apego preocupado (32.1\%) y casos inclasificables (32.1\%).

A fin de evaluar si existe asociación entre depresión y estilo de apego, se ocupó el estadístico Chi-cuadrado $\left(\chi^{2}\right)$. Los análisis indican que existe una asociación significativa entre ambas variables: $\chi^{2}(3, N=56)=$ $20.33 p<.01$.

Específicamente, de los resultados se desprende que los porcentajes de estilos de apego seguro e inseguro difieren entre ambos grupos de mujeres. La proporción de estilos de apego seguro es más alta en el grupo de mujeres sin depresión, mientras que en el grupo de mujeres deprimidas hay una proporción mayor de apegos preocupados e inclasificables.

A fin de evaluar en qué escalas del Camir existen diferencias entre ambos grupos evaluados (mujeres con y sin depresión), se realizó un análisis con la prueba $t$ para muestras independientes, asumiendo varianzas homogéneas de acuerdo al test de Levene $(p>.05)$. Los resultados señalan que existen diferencias estadísticamente significativas en las escalas de interferencia parental, $t(54)=-5.17, p<.05$, preocupación familiar, $t(54)=4.87, p<.05$, resentimiento de infantilización, $t(54)=3.93, p<.05$, apoyo familiar, $t(54)=4.97, p<$ .05 , distancia familiar, $t(54)=3.88, p<.05$, resentimiento de rechazo, $t(54)=4.44, p<.05$, traumatismo parental, $t(54)=2.0, p<.05$, dimisión parental, $t(54)=-4.29, p<$ .05 , y valoración de la jerarquía, $t(54)=12.79, p<.05$. Específicamente, las mujeres con depresión presentan medias más altas que las mujeres sin depresión en todas las escalas antes mencionadas, salvo en las escalas de interferencia parental y dimisión parental donde son estas últimas quienes presentan promedios más elevados (ver Tabla 1).

\section{Discusión}

Esta investigación tuvo como objetivo describir los estilos de apego de un grupo de mujeres con diagnóstico de depresión y compararlas con una muestra no clínica. Considerando los resultados obtenidos, es posible afirmar que existen diferencias significativas en los patrones de apego en ambos grupos estudiados. En la muestra de pacientes con depresión, la mayoría de las mujeres presentaron apego inseguro (39.2\%) e inclasificable (32.1\%), siendo más predominante el apego preocupado (32.1\%) que el apego rechazante $(7.1 \%)$. En este sentido, considerando la asociación entre depresión y estilo de apego, los análisis permitieron comprobar que existe una asociación significativa entre ambas variables, presentándose diferencias en los porcentajes de patrones de apego seguro e inseguro entre ambos grupos estudiados. El estilo de apego seguro fue reportado en mayor medida por el grupo de población no clínica (85.7\%); los estilos de apego inseguro e inclasificable, en cambio, son más frecuentemente reportados por las mujeres con diagnóstico de depresión que por las que no tienen. En el caso del apego rechazante, las mujeres sin depresión presentan un 3.6\% versus un $7.1 \%$ en las mujeres con depresión; en el apego preocupado, mujeres sin depresión presentan un $10.7 \%$ versus un $32.1 \%$ en aquellas que tienen el diagnóstico y, por último, los casos inclasificables sólo se presentan en la muestra con depresión (32.1\%). La alta presencia de estos casos, que se caracterizan por presentar altos puntajes tanto en preocupación como en evitación, puede explicarse por las características de la población investigada, ya que la totalidad presentaba el diagnóstico de episodio depresivo grave y es, además, coherente con los resultados de otras investigaciones internacionales en muestras clínicas (Miljkovitch et al., 2005; Pierrehumbert, Bader, Miljkovitch, Mazet, Amar y Halfon, 2002). En este contexto, sería interesante la utilización de instrumentos como la $A A I$, que permitieran la obtención de la categoría "no resuelto/desorganizado" o "no clasificado", dado que ambas categorías se han asociado con desórdenes psiquiátricos (Hesse, 1999; Lecannelier, Ascanio, Flores y Hoffman, 2011) y que, en el caso de instrumentos como Camir, no permiten la obtención de la categoría "no resuelto/desorganizado", aun cuando se puede hipotetizar que los casos inclasificables presentan dificultades en una organización adecuada de las estrategias de apego, sumado a la alta preocupación y evitación.

Los resultados del presente estudio confirman, al igual que diversas investigaciones nacionales (Farkas et al., 2008; 
Santelices et al., 2009; Santelices et al., 2011) e internacionales (Beatson y Taryan, 2003; Carnelley et al., 1994; Conradi y De Jonge, 2009; Cooley et al., 2010; Graham, 2000; Haaga et al., 2002; Ivarsson et al., 2010; Kidd y Sheffield, 2005; Kuscu et al., 2008; Liu et al., 2009; Mak et al., 2010; Miljkovitch et al., 2005; Muris et al., 2000; Myhr et al., 2004; Simonelli et al., 2004; Surcinelli et al., 2010; Wearden et al., 2005; Wilkinson y Mulcahy, 2010; Zaghdoudi et al., 2009), que un estilo de apego seguro actúa como factor protector en el desarrollo de la depresión y la sintomatología depresiva, y que los estilos inseguros se constituyen en un factor de vulnerabilidad.

Si se examina un nivel de análisis más específico, es interesante subrayar la alta predominancia del estilo preocupado en las mujeres que presentan depresión, más aún, considerando que es la primera investigación realizada en Chile acerca de la relación entre los estilos de apego adulto y la depresión, en muestra clínica propiamente tal. En investigaciones internacionales realizadas con muestras clínicas, utilizando la clasificación de Bartholomew y Horowitz (1991), la mayor vulnerabilidad a la depresión se ha reportado en los estilos temerosos y preocupados (Haaga et al., 2002), aunque algunos autores enfatizan una relación más estrecha con el estilo temeroso (Carnelley et al., 1994; Conradi y De Jonge et al., 2009; Meredith et al., 2007); algo similar ocurre en muestras no clínicas, asociando estilos temerosos y preocupados con mayor sintomatología depresiva (Carnelley et al., 1994; Cooley et al., 2010; Permuy et al., 2009; Simonelli et al., 2004; Surcinelli et al., 2010; Van Buren y Cooley, 2002). Lo anterior refleja importantes coincidencias entre la presente investigación y lo reportado en los estudios internacionales señalados, sobre todo si se considera que algunos autores establecen que los estilos preocupados y temerosos podrían ser clasificados como ansiosos / ambivalentes (Simonelli et al., 2004), categoría equivalente al estilo preocupado de la clasificación adulta tradicional (Pierrehumbert et al., 1996) utilizada en la presente investigación. Si además se considera que la rumiación es una de las estrategias de regulación emocional características del estilo de apego ambivalente (Garrido, 2006) y que investigaciones chilenas han dado cuenta de la asociación entre rumiación negativa y síntomas depresivos (Cova, Rincón y Melipillán, 2009), los datos del presente estudio son también coherentes desde esta perspectiva.

Estos resultados tienen implicancias importantes para el desarrollo de intervenciones dirigidas a la prevención y tratamiento de esta patología, de alta prevalencia en Chile y el mundo; por un lado, el desarrollo e implementación de iniciativas que potencien un estilo de apego seguro puede implicar un factor claramente protector en la aparición de esta patología, disminuyendo la vulnerabilidad ante ésta $y$, por otro lado, en el caso que las personas ya presenten la enfermedad, es posible generar intervenciones que consideren aspectos relevantes acerca del estilo de apego del sujeto, de tal modo que las estrategias específicas sean elaboradas a partir del perfil de cada paciente. De tal forma, una posibilidad a evaluar en contextos de salud, ya sea de atención primaria, secundaria o terciaria, sería la instauración de sistemas de pesquisa temprana de estilos de apego preocupado y rechazante en consultantes, que permitiera una actuación más expedita en tales casos, considerando la mayor complejidad que éstos pueden implicar. En este sentido, el presente estudio se constituye en un aporte para la realidad nacional, confirmando en ésta aspectos ampliamente señalados a nivel internacional. Permite, además, agregar una nueva mirada analítica, que entrega mayor profundidad en el análisis de esta patología y, por lo mismo, una propuesta de tratamiento más integral.

La investigación realizada presenta una serie de limitaciones a considerar. En primer lugar, el tamaño de la muestra era pequeño, aun cuando en muestras clínicas suele ser mayor la dificultad en la inclusión de los casos. Por otro lado, la utilización de un instrumento de autoinforme como única evaluación, que requiere una cierta capacidad de instrospección, puede implicar sesgos en las respuestas de los sujetos, quienes pueden tender a negar o exagerar los problemas, no permitiendo así un reconocimiento adecuado del estilo en cuestión; en este sentido, algunos autores enfatizan la necesidad de considerar las limitaciones de mediciones particulares, de realizar validaciones sistemáticas de las mediciones de apego y utilizar la mayor cantidad posible de indicadores o realizar mediciones múltiples (Bartholomew, 1994; Bifulco, 2002; Stein et al., 1998).

\section{Referencias}

Ainsworth, M., Blehar, M., Waters, E. y Wall, S. (1978). Patterns of attachment: A psychological study of the strange situation. Hillsdale: Erlbaum.

Altin, M. y Terzi, S. (2010). How does attachment styles relate to intimate relationship to aggravate the depressive symptoms?. Procedia Social and Behavioral Sciences, 2, 1008-1015.

Armour, C., Elklit, A. y Shevlin, M. (2011). Attachment typologies and posttraumatic stress disorder (PTSD), depression and anxiety: a latent profile analysis approach. European Journal of Psychotraumatology, 2, 1-9.

Bartholomew, K. (1994). Assessment of individual differences in adult attachment. Psychological Inquiry, 5, 23-67.

Bartholomew, K. y Horowitz, L. (1991). Attachment styles among young adults: a test of a four-category model. Journal of Personality and Social Psychology, 61, 226-244. 
Beatson, J. y Taryan, S. (2003). Predisposition to depression: the role of attachment. Australian and New Zealand Journal of Psychiatry, 37, 219-225.

Berman, W. y Sperling, M. (1994). The structure and function of adult attachment. En M. Sperling y W. Berman (Eds.), Attachment in Adults. Clinical and Developmental Perspectives (pp. 3-28). New York: Guilford Press.

Besser, A. y Priel, B. (2008). Attachment, depression and fear of death in older adults: the roles of neediness and perceived availability of social support. Personality and Individual Differences, 44, 1711-1725.

Bifulco, A. (2002). Attachment style measurement: A clinical and epidemiological perspective. Attachment y Human Development, 4, 180-188.

Bifulco, A., Kwon, J., Jacobs, C., Moran, P. Bunn, A. y Beer, N. (2006). Adult attachment style as mediator between childhood neglect/abuse and adult depression and anxiety. Social Psychiatry and Psychiatric Epidemiology, 41, 796-805.

Bifulco, A., Moran, P., Ball, C. y Bernazzani, O. (2002a). Adult attachment style. I: its relationship to clinical depression. Social Psychiatry and Psychiatric Epidemiology, 37, 50-59.

Bifulco, A., Moran, P., Ball, C. y Lillie, A. (2002b). Adult attachment style. II: its relationship to psychosocial depressive-vulnerability. Social Psychiatry and Psychiatric Epidemiology, 37, 60-67.

Bowlby, J. (1969/1998). El apego y la pérdida: Vol. 1. El apego (1ª Reimpresión). Barcelona: Paidós.

Bowlby, J. (1973/1998). El apego y la pérdida: Vol. 2. La separación (3 ${ }^{\mathrm{a}}$ Reimpresión). Barcelona: Paidós.

Bowlby, J. (1980/1993). El apego y la pérdida: Vol. 3. La pérdida afectiva ( $1^{\mathrm{a}}$ Reimpresión). Buenos Aires: Paidós.

Bowlby, J. (1986). Vinculos afectivos: Formación, desarrollo y pérdida. Madrid: Morata.

Capozzi, F., Casini, M., Romani, M., De Gennaro, L., Nicolais, G. y Solano, L. (2008). Psychiatric comorbidity in learning disorder: analysis of family variables. Child Psychiatry and Human Development, 39, 101-110.

Carnelley, K., Pietromonaco, P. y Jaffe, K. (1994). Depression, working models of others and relationship functioning. Journal of Personality and Social Psychology, 66, 127-140.

Conradi, H. y De Jonge, P. (2009). Recurrent depression and the role of adult attachment: a prospective and a retrospective study. Journal of Affective Disorders, 116, 93-99.

Cooley, E., Van Buren, A. y Cole, S. (2010). Attachment styles, social skills and depression in collage women. Journal of College Counseling, 13, 50-62.

Cova, F., Rincón, P. y Melipillán, R. (2009). Reflexión, rumiación negativa y desarrollo de sintomatología depresiva en adolescentes de sexo femenino. Terapia Psicológica, 27, 155-160.

Davidson, C., Wingate, L., Grant, D., Judah, M. y Mills, A. (2011). Interpersonal suicide risk and ideation: the influence of depression and social anxiety. Journal of Social and Clinical Psychology, 30, 842-855.

Dozier, M., Stovall, C. y Albus, K. (1999). Attachment and psychopathology in adulthood. En J. Cassidy y P. Shaver (Eds.), Handbook of attachment (pp. 497-519). New York: Guilford Press.

Evans, L. y Wertheim, E. (2005). Attachment styles in adult intimate relationships: comparing women with bulimia nervosa symptoms, women with depression and women with no clinical symptoms. European Eating Disorders Review, 13, 285-293.

Farkas, Ch., Santelices, M. P., Aracena, M. y Pinedo, J. (2008). Apego y ajuste socioemocional: un estudio en embarazadas primigestas. Psykhe, $17,65-80$

Feeney, J., Noller, P. y Hanrahan, M. (1994). Assessing adult attachment. En M. Sperling y W. Berman (Eds.), Attachment in adults. Clinical and developmental perspectives (pp. 128-152). New York: Guilford Press.

Figueiredo, B., Bifulco, A., Pacheco, A., Costa, R. y Magarinho, R. (2006). Teenage pregnancy, attachment style and depression: a comparison of teenage and adult pregnant women in a Portuguese series. Attachment y Human Development, 8, 123-138.

Gamble, S. y Roberts, J. (2005). Adolescents' perceptions of primary caregivers and cognitive style: the roles of attachment security and gender. Cognitive Therapy and Research, 29, 123-141.
Garrido, L. (2006). Apego, emoción y regulación emocional. Implicaciones para la salud. Revista Latinoamericana de Psicología, 38, 493-507.

Garrido, L., Santelices, M. P., Pierrehumbert, B. y Armijo, I. (2009). Validación chilena del instrumento para evaluar apego en el adulto, CAMIR. Revista Latinoamericana de Psicología, 41, 81-98.

Garrido, L. (2013). Cuestionario de evaluación de apego en el adulto, CaMir (Cartes, Modèles individuels de relation): Normas para aplicar, tabular e interpretar. Talca: Colección Cuadernos Académicos UC Maule.

González, L. y Méndez, L. (2006). Relación entre autoestima, depresión y apego en adolescentes urbanos de la comuna de Concepción, Chile. Terapia Psicológica, 24, 5-14.

Graham, C. (2000). School-aged children's vulnerability to depressive symptomatology: the role of attachment security, maternal depressive symptomatology and economic risk. Development and Psychopathology, $12,201-213$

Greenberg, M. (1999). Attachment and psychopathology in childhood. En J. Cassidy y P. Shaver (Eds.), Handbook of attachment (pp. 469-496). New York: Guilford Press.

Griffin, D. y Bartholomew, K. (1994). The metaphysics of measurement: The case of adult attachment. En K. Bartholomew y D. Pealman (Eds.), Advances in Personal Relationships, 5, 17-52. London: Kingsley.

Haaga, D., Yarmus, M., Hubbard, S., Brody, C., Solomon, A., ... Kirk, L. (2002). Mood dependency of self-rated attachment style. Cognitive Therapy and Research, 26, 57-71.

Hankin, B. (2005). Childhood maltreatment and psychopathology: prospective tests of attachment, cognitive vulnerability and stress as mediating processes. Cognitive Therapy and Research, 29, 645-671.

Harris, T. (2002). Attachment-related psychodynamics: Another shake to the kaleidoscope. Attachment and Human Development, 4, 201-206.

Hazan, C. y Shaver, P. (1987). Romantic love conceptualized as an attachment process. Journal of Personality and Social Psychology, 52, 511-524.

Hazan, C. y Shaver, P. (1994). Deeper into attachment theory. Psychological Inquiry, 5, 68-79.

Heerlein, A. (2000). Trastornos del ánimo. En A. Heerlein (Ed.), Psiquiatría Clínica (pp. 313-341). Santiago: Sociedad de Neurología, Psiquiatría y Neurocirugía.

Hesse, E. (1999). The adult attachment interview. Historical and current perspectives. En J. Cassidy y P. Shaver (Eds.), Handbook of attachment (pp. 395-433). New York: Guilford Press.

Hortacsu, N., Cesur, S. y Oral, A. (1993). Relationships between depression and attachment styles in parent -and institution- reared turkish children. The Journal of Genetic Psychology 154, 329-337.

Ivarsson, T., Granqvist, P., Gillberg, C. y Broberg, A. (2010). Attachment states of mind in adolescents with obsessive-compulsive disorder and or depressive disorders: a controlled study. European Child y Adolescent Psychiatry, 19, 845-853.

Jacobvitz, D., Curran, M. y Moller, N. (2002). Measurement of adult attachment: The place of self-report and interview methodologies. Attachment y Human Development, 4, 207-215.

Kerns, K., Brumariu, L. y Seibert, A. (2011). Multi-method assessment of mother-child attachment: links to parenting and child depressive symptoms in middle childhood. Attachment y Human Development, 13, 315-333.

Kidd, T. y Sheffield, D. (2005). Attachment style and symptom reporting: examining the mediating effects of anger and social support. British Journal of Health Psychology, 10, 531-541.

Kuscu, M., Akman, I., Karabekiroglu, A., Yurdakul, Z., Orhan, L., ... Ozdemir, N. (2008). Early adverse emocional response to childbirth in Turkey: the impact of maternal attachment styles and family support. Journal of Psychosomatic Obstetrics y Gynecology, 29, 33-38.

Lecannelier, F., Ascanio, L., Flores, F. y Hoffman, M. (2011). Apego y psicopatología: una revisión actualizada sobre los modelos etiológicos parentales del apego desorganizado. Terapia Psicológica, 29, 107-116.

Lecannelier, F., Undurraga, V., Olivares, A., Rodríguez, J., Núñez, J., Hoffmann, M., Goldstein, N. y Larraín, C. (2009). Estudio de eficacia sobre dos intervenciones basadas en el fomento del apego temprano en díadas madre-bebé provenientes de la Región Metropolitana en Santiago de Chile. Revista Argentina de Clínica Psicológica, XVIII, 143-155. 
Li, T., Li, J. y Dai, Q. (2008). Adult attachment, social support, and depression level of poststroke patients. Social Behavior Personality, 36, 1341-1352.

Liu, Q., Nagata, T., Shono, M. y Kitamura, T. (2009). The effects of adult attachment and life stress on daily depression: a sample of japanese university students. Journal of Clinical Psychology, 65, 639-652.

Main, M. (2000). The organized categories of infant, child, and adult attachment: flexible vs. inflexible attention under attachment-related stress. Journal of the American Psychoanalytic Association, 48, 1055-1096.

Micin, S. y Bagladi, V. (2011). Salud mental en estudiantes universitarios: incidencia de psicopatología y antecedentes de conducta suicida en población que acude a un servicio de salud estudiantil. Terapia Psicológica, 29, 53-64.

Main, M. y Solomon, J. (1990). Procedures for identifying infants as disorganized/disoriented during Ainsworth strange situation. En M. Greenberg, D. Cicchetti y M. Cummings (Eds.). Attachment in the preschool years: theory, research and intervention (pp. 121-160). Chicago: University of Chicago Press.

Mak, M., Simpson, J. y Rholes, W. (2010). Adult attachment, perceived support, and depressive symptoms in chinese and American cultures. Journal of Social and Clinical Psychology, 29, 144-165.

Martínez, C. y Núñez, C. (2007). Entrevista de Prototipos de Apego Adulto (EPAA): propiedades psicométricas de su versión en Chile. Revista Interamericana de Psicología, 41, 261-274.

Martínez, C. y Santelices, M. P. (2005). Evaluación del apego en el adulto: una revisión. Psykhe, 14, 181-191.

Melis, F., Dávila, M., Ormeño, V., Vera, V., Greppi, C. y Gloger, S. (2001). Estandarización del P.B.I. (Parental Bonding Instrument), versión adaptada a la población entre 16 y 64 años del Gran Santiago. Revista Chilena de Neuropsiquiatría, 39, 132-139.

Meredith, P., Strong, J. y Feeney, J. (2007). Adult attachment variables predict depression before and after treatment for chronic pain. European Journal of Pain, 11, 164-170.

Mikulincer, M. y Shaver, P. (2007). Attachment in adulthood. Structure, Dynamics and Change. New York: The Guilford Press.

Ministerio de Salud, División de Salud de las Personas (1999). Las enfermedades mentales en Chile: magnitud y consecuencias. Santiago: Autor.

Ministerio de Salud. (2003). Resultados I Encuesta de Salud, Chile 2003. Santiago: Autor.

Ministerio de Salud (2010). Encuesta Nacional de Salud ENS, Chile 20092010. Santiago: Autor.

Ministerio de Salud (2011). Estrategia Nacional de Salud para el Cumplimiento de los Objetivos Sanitarios de la Década 2011-2020. Santiago: Autor.

Miljkovitch, R., Pierrehumbert, B., Karmaniola, A., Bader, M. y Halfon, O. (2005). Assessing attachment cognitions and their associations with depression in youth with eating or drug misuse disorders. Substance Use y Misuse, 40, 605-623.

Monk, C., Leight, K. y Fang, Y. (2008). The relationship between women's attachment style and perinatal mood disturbance: implications for screening and treatment. Archives of Womens Mental Health, $11,117-129$

Morley, T. y Moran, G. (2011). The origins of cognitive vulnerability in early childhood: mechanisms linking early attachment to later depression. Clinical Psychology Review, 31, 1071-1082.

Muris, P., Mayer, B. y Meesters, C. (2000). Self-reported attachment style, anxiety, and depression in children. Social Behavior and Personality, $28,157-162$.

Myhr, G., Sookman, D. y Pinard, G. (2004). Attachment security and parental bonding in adults with obsessive-compulsive disorder: a comparison with depressed out-patients and healthy controls. Acta Psychiatrica Scandinavica, 109, 447-456.

Nishikawa, S., Hägglöf, B. y Sundbom, E. (2010). Contributions of attachment and self-concept on internalizing and externalizing problems among Japanese adolescents. Journal of Child and Family Studies, 19, 334-342.

Organización Mundial de la Salud (1999). Clasificación Internacional de Enfermedades CIE 10: Trastornos Mentales y del Comportamiento: Descripciones Clínicas y Pautas para el Diagnóstico. Ginebra: Meditor.
Permuy, B., Merino, H. y Fernández-Rey, J. (2009). Adult attachment styles and cognitive vulnerability to depression in a sample of undergraduate students: the mediational roles of sociotropy and autonomy. International Journal of Psychology, 1-7.

Pesonen, A., Räikkönen, K., Strandberg, T., Keltikangas-Järvinen, L. y Järvenpää, A. (2004). Insecure adult attachment style and depressive symptoms: implications for parental perceptions of infant temperamento. Infant Mental Health Journal, 25, 99-116.

Pierrehumbert, B., Bader, M., Miljkovitch, R., Mazet, P., Amar, M. y Halfon, O. (2002). Strategies of emotion regulation in adolescents and young adults with substance dependence or eating disorders. Clinical Psychology and Psychotherapy, 9, 384-394.

Pierrehumbert, B., Karmaniola, A., Sieye, A., Meister, C., Miljkovitch, R. y Halfon, O. (1996). Les modèles de relations: Développement d'un autoquestionnaire d' attachement pour adultes. Psychiatrie de $L$ Enfant, 1, 161-206.

Preti, A., Tondo, L., Sisti, D., Rocchi, M. y De Girolamo, G. (2010). Correlates and antecedents of hospital admission for attempted suicide: a nationwide survey in Italy. European Archives of Psychiatry and Clinical Neuroscience, 260, 181-190.

Quezada, V. y Santelices, M. P. (2010). Apego y psicopatología materna: relación con el estilo de apego del bebé al año de vida. Revista Latinoamericana de Psicología, 42, 53-61.

Retamal, P., Florenzano, R., Mourgues, C., Martínez, X., Chamorro, X., ... Leighton, C. (2004). Programa Nacional de Diagnóstico y Tratamiento de la Depresión Severa. Santiago: Universidad de Chile, Escuela de Medicina.

Richards, D. (2011). Prevalence and clinical course of depression: a review. Clinical Psychology Review, 31, 1117-1125.

Rivera, D., Cruz, C. y Muñoz, C. (2011). Satisfacción en las relaciones de pareja en la adultez emergente: el rol del apego, la intimidad y la depresión. Terapia Psicológica, 29, 77-83.

Roelofs, J., Meesters, C., Huurne, M., Bamelis, L. y Muris, P. (2006). On the links between attachment style, parental rearing behaviours, and internalizing and externalizing problems in non-clinical children. Journal of Child and Family Studies, 15, 331-344.

Roisman, G., Holland, A., Fortuna, K., Fraley, C., Clausell, E. y Clarke, A. (2007). The adult attachment interview and self-reports of attachment style: an empirical rapprochement. Journal of Personality and Social Psychology, 92, 678-697.

Ruijten, T., Roelofs, J. y Rood, L. (2011). The mediating role of rumination in the relation between quality of attachment relations and depressive symptoms in non-clinical adolescents. Journal of Child and Family Studies, 20, 452-459.

Santelices, M. P., Carvacho, C., Farkas, Ch., León, F., Galleguillos, F. e Himmel, E. (2012). Medición de la sensibilidad del adulto con niños de 6 a 36 meses de edad: construcción y análisis preliminares de la Escala de Sensibilidad del Adulto, E.S.A. Terapia Psicológica, 30, 19-29.

Santelices, M. P., Garrido, L., Fuentes, M., Guzmán, M. y Lizano, M. (2009). Estudio de los estilos de apego adulto en un grupo de pacientes con insuficiencia renal crónica terminal. Universitas Psychologica, 8, 413-422.

Santelices, M. P., Guzmán, M. y Garrido, L. (2011). Apego y psicopatología: Estudio comparativo de los estilos de apego en adultos con y sin sintomatología ansioso-depresiva. Revista Argentina de Clínica Psicológica, $X X, 49-55$.

Shaver, P. y Mikulincer, M. (2002). Dialogue on adult attachment: diversity and integration. Attachment y Human Development, 4, 243-247.

Simonelli, L., Ray, W. y Pincus, A. (2004). Attachment models and their relationships with anxiety, worry and depression. Counseling and Clinical Psychology Journal, 1, 107-118.

Spencer, R., Alonso-Arbiol, I. y Fresno, A. (2013). Romantic attachment in Chilean adults: development of a short-form version of the experiences in close relationships. Spanish Journal of Psychology, 16, 1-9.

Spencer, R., Guzmán, M., Fresno, A. y Ramos, N. (2013). Validación chilena del cuestionario de evaluación del apego romántico Experiences in close relationships (ECR): análisis de la validez de criterio. Terapia Psicológica, 31, 313-324. 
Stein, H., Jacobs, N., Ferguson, K., Allen, J. y Fonagy, P. (1998). What do adult attachment scales measure?. Bulletin of the Menninger Clinic, 62, 33-81.

Strodl, E. y Noller, P. (2003). The relationship of adult attachment dimensions to depression and agoraphobia. Personal Relationships, 10, 171-185.

Surcinelli, P., Rossi, N., Montebarocci, O. y Baldaro, B. (2010). Adult attachment styles and psychological disease: examining the mediating role of personality traits. The Journal of Psychology, 144, 523-534.

Tasca, G., Szadkowski, L., Illing, V., Trinneer, A., Grenon, R., ... Demidenko, N. (2009). Adult attachment, depression, and eating disorder symptoms: the mediating role of affect regulation strategies. Personality and Individual Differences, 47, 662-667.

Van Buren, A. y Cooley, E. (2002). Attachment styles, view of self and negative affect. North American Journal of Psychology, 4, 417-430.

Whiffen, V., Kerr, M. y Kallos-Lilly, V. (2005). Maternal depression, adult attachment and children's emotional distress. Family Process, 44, 93-103.

Wilkinson, R. y Mulcahy, R. (2010). Attachment and interpersonal relationships in postnatal depression. Journal of Reproductive and Infant Psychology, 28, 252-265.

Zaghdoudi, L., Moubarkim, A., Hlayem, S., Bechir, M. y Labbane, R. (2009). Relation between styles d' attachement, perception du sotien social et depression. Annales Médico Psychologiques, 167, 657-661. 
